\section{AGORA DÉBATS/JEUNESSES}

Appel à propositions d'articles pour la revue

\author{
Agora débats/jeunesses n89 (2021/3)
}

\title{
Des professionnels pour les jeunes
}

Dossier thématique coordonné par Valérie Becquet, professeure des universités, CY Cergy Paris Université, EMA et Martial Meziani, maitre de conférences, CY Cergy Paris Université, EMA

La catégorie des «professionnels de la jeunesse » ou, pour reprendre la traduction française de "youth worker " utilisée au niveau européen, des "travailleurs de jeunesse ", s'est progressivement constituée au milieu du $\mathrm{XIX}^{\mathrm{e}}$ siècle en lien avec la structuration des mouvements de jeunesse et d'éducation populaire d'une part, et avec l'émergence d'une action publique en direction des jeunes, d'autre part. Quatre grands moments renvoyant à des systèmes de valeurs distincts seraient ainsi repérables (Loncle, 2003). Dans un premier temps (entre 1870 et 1936), l'action publique est principalement portée au niveau local par des œuvres, des associations et des municipalités et concerne les questions éducatives, sanitaires et sociales. Au niveau national, ce sont l'instruction et la conscription qui structurent l'intervention de l'État. Dans un deuxième temps (entre 1936 et 1983), avec l'avènement de l'État-Providence, l'État joue un rôle plus important avec l'élaboration des orientations et des réglementations en matière d'encadrement des enfants et des jeunes, la création des équipements de loisirs et l'organisation des relations avec les collectivités territoriales et les mouvements de jeunesse, dont la place évolue au cours de cette période. Dans un troisième temps (entre 1983 et 1995), la territorialisation de l'action publique est encouragée par l'État à travers les politiques de la ville et de l'éducation prioritaire (Avenel, 2010 ; Donzelot, 2003 ; Tissot, 2007 ; Van Zanten, 2004). Elle résulte aussi de l'initiative des collectivités territoriales et entraîne le développement des délégations à la jeunesse dans les municipalités (Loncle, 2011). En résulte une fragmentation de l'action correspondant aux difficultés rencontrées par les jeunes (insertion sociale et professionnelle, par exemple) ou aux problèmes que posent les jeunes à la société (lutte contre la délinquance, par exemple). Enfin, à partir du milieu des années 1990, y compris dans les secteurs les plus centralisés, l'action publique se caractérise par une approche multiple: multi-acteurs, multi-niveaux et multi-secteurs, ce qui rend son appréhension difficile.

Cette structuration progressive d'une action publique autour des publics juvéniles a conduit à une professionnalisation des acteurs chargés de la concevoir et de la mettre en œuvre. Elle s'est opérée en plusieurs étapes : à partir de la première moitié $d u x{ }^{e}$ siècle à travers l'organisation du secteur de l'intervention sociale, des loisirs et des sports et la création des métiers d'éducateurs spécialisés et d'animateurs ; puis, progressivement, en lien avec des politiques plus sectorielles destinées à prendre en charge des problématiques spécifiques aux différents échelons territoriaux. Cette professionnalisation ne résulte pas uniquement de l'action des pouvoirs publics : si ces derniers ont contribué à la définition des contours d'une pluralité d'activités, que ce soit à travers les référentiels de compétences, les certifications (Brunel, 2015) ou encore les dispositifs ciblés, les mouvements et les associations de jeunesse et d'éducation populaire, de sport ou d'intervention sociale, entre autres, l'ont dès 
le départ influencé et jouent encore en tant qu'employeurs un rôle actif dans l'institutionnalisation de ces activités. D'ailleurs, que ce soit du côté de l'animation volontaire, puis professionnelle, ou du côté de l'éducation spécialisée, l'histoire est émaillée de crises, de luttes de pouvoir et de tactiques plus quotidiennes autour de la définition des missions, des compétences, des certifications, etc. (Camus, 2008 ; Camus et Lebon, 2015 ; Lebon, 2009 ; Richelle, Ziegelmeyer et Rubi, 2013).

II reste que, compte-tenu du contexte socio-économique, des dynamiques politiques, des réformes territoriales redéployant la "compétence jeunesse " et de la diversification des formes d'instrumentation des questions de jeunesse (Becquet, 2018 ; Halpern, Lascoumes et Le Galès, 2014), les frontières de la catégorie "professionnels de la jeunesse » apparaissent de plus en plus incertaines. D'ailleurs, les débats européens le reflètent. Ainsi, la définition retenue par le Conseil de l'Europe témoigne de l'étendue des activités et de la variété des acteurs, en particulier en incluant à la fois les salariés et les bénévoles, tout en réaffirmant des principes d'intervention anciens comme la participation des jeunes: "Le travail de jeunesse est un concept large qui couvre un vaste éventail d'activités sociales, culturelles, éducatives, environnementales et/ou de nature politique par, avec et pour les jeunes, en groupe ou individuellement. Le travail de jeunesse est assuré par des travailleurs de jeunesse rémunérés et bénévoles et repose sur un processus d'apprentissage non formels et informels axés sur les jeunes et sur la participation volontaire ${ }^{1}$. " Quant aux récents textes adoptés dans le cadre de la nouvelle stratégie de l'Union européenne en faveur de la jeunesse pour la période 2019-2027, ils font du "travail socio-éducatif » un levier pour soutenir l'autonomisation des jeunes: "Le travail socio-éducatif apporte des avantages uniques aux jeunes en transition vers l'âge adulte ; il leur offre un environnement sûr qui leur permet de gagner en confiance et d'apprendre de façon non formelle. [...] Dans certains cas, le travail socio-éducatif fait le pont vers l'éducation, la formation ou le travail, empêchant ainsi l'exclusion. ${ }^{2}$ " À quels acteurs renvoient ces définitions en France ? Quels seraient les travailleurs, voire les bénévoles qu'inclut le Conseil de l'Europe, dont les pouvoirs publics devraient soutenir « la qualité, l'innovation et la reconnaissance » du travail effectué ? À bien des égards et sans aller au-delà des textes les plus récents, les discours des institutions européennes rappellent combien le "travail de jeunesse " ou le "travail socio-éducatif » renvoie à une pluralité d'acteurs.

En fait, cet ensemble est à la fois défini par des secteurs d'intervention (social, socioéducatif, éducation, insertion, justice, etc.), par des destinataires (adolescents, jeunes, jeunes en difficultés, jeunes placés, etc.), par des fonctions (le contrôle, la prévention, l'éducation, l'insertion, l'accompagnement, l'autonomisation, etc.) ou encore par la pluralité des acteurs publics et privés situés aux différents échelons territoriaux. Les visées et les contours des activités, tout comme les contextes d'exercice, de ces professionnels seraient également directement impactés par les mutations organisationnelles engendrées par la Nouvelle Gestion Publique (NGP), dont résulteraient des paradoxes et des tensions (Demazière, Lessard et Morrisette, 2013) : entre logiques d'action gestionnaire et techniciste et logiques éducative et sociale (Chantraine et Sallée, 2013 ; Labadie et Crochu, 2018a et 2018b) ; entre geste professionnel et action militante (Poujol, 1989 ; Ravon et Ion, 2012 ) ; entre accompagnement individualisé (coaching) et intervention collective (empowerment) ;

\footnotetext{
1 https://www.coe.int/fr/web/youth/youth-work

2 Mobiliser, connecter et autonomiser les jeunes : une nouvelle stratégie de l'Union européenne en faveur de la jeunesse. Communication de la Commission au Parlement européen, au Conseil européen, au Conseil, au Comité économique et sociale européen et au Comité des régions, Bruxelles, 22 mai 2018.
} 
entre relation éducative et relation "client »; ou encore entre injonction à l'innovation et standardisation des "bonnes pratiques" (Broudic, 2018). In fine, se jouent des formes d'articulation, d'hybridation ou des oppositions entre des logiques relatives à la NGP et d'autres qui peuvent être soit plus anciennes, relevant du suivi personnalisé (Rosanvallon, 1995 ; Astier, 2000), soit plus récentes et relevant de l'éthique du care (Gilligan, 1982 ; Tronto, 1993 ; Paperman et Laugier, 2005) et de son institutionnalisation (Pattaroni, 2005 ; Meziani, 2018).

De manière schématique, se côtoieraient actuellement, d'un côté, des professionnels dits "établis ", c'est-à-dire des acteurs historiquement constitués, dont les modalités de qualification et de recrutement sont règlementées et les activités inscrites dans un espace politico-administratif relativement balisé (comme les éducateurs spécialisés ou les animateurs) (Falchun, Robène et Terret, 2016 ; Lebon, 2006, 2014 ; Lebon et Lescure, 2006 ; Sallée, 2007, 2014) et, de l'autre, des professionnels dits "émergents » ou "nouveaux », c'est-à-dire des acteurs produits par l'action publique, dont les compétences, les modes d'accès au marché du travail et les activités sont pluriels et fluctuants (Muniglia et Thalineau, 2012; Trindade-Chadeau, 2012). Ces derniers portent des appellations relativement diverses, comme coordinateur, chargé de projet, chargé de mission, référent, médiateur, etc., et ne sont pas constitués en groupe professionnel. Leur activité privilégie la polyvalence et prend appui sur des professionnalités plurielles. Ils peuvent être qualifiés de professionnels d'interface (interfaces sectorielle, institutionnelle et fonctionnelle). Enfin, un dernier groupe de professionnels semble à mi-chemin entre ces deux types de professionnels. En effet, certains métiers, relativement récents, tels que les arts-thérapeutes ou les coachs, peuvent être constitués en collectif afin d'accroître leur légitimité ou inscrits dans des réseaux reconnus comme ayant des compétences particulières. Si ces métiers relèvent des professions libérales, ils tendent à s'organiser comme certains intervenants afin de favoriser l'employabilité, par exemple, ou comme des professionnels paramédicaux.

Les constats qui précèdent invitent à s'émanciper de la catégorie de « professionnels de la jeunesse ", dont il est aisé de saisir le caractère illusoire et, par conséquent, peu heuristique pour l'analyse. Ce dossier s'intéresse aux professionnels qui gravitent autour des jeunes en dehors du champ scolaire. En effet, si les travaux concernant l'école pointent à quel point les acteurs scolaires sont affectés dans leurs prérogatives et leur activités professionnelles par les différentes réformes du système scolaire en ce qui concerne les frontières entre l'école et le hors école (Moignard, 2018 : Moignard et Rubi, 2013), il apparaît que les autres champs sont impactés de manière spécifique, les enjeux politiques et économiques variant selon les espaces sociaux en question. Afin de favoriser la discussion sur la situation et les évolutions actuelles et de donner à voir la variété des secteurs et des contextes d'exercice concernés, ce dossier privilégiera l'analyse des activités et des professionnalités. Comment s'élaborentelles et à quelles logiques politiques et techniques répondent-elles ? Quels sont les effets des contextes d'exercice qu'ils soient orientés par les échelons territoriaux et les secteurs d'intervention, mais aussi par les types d'employeurs, les dispositifs de référence, la prise en charge de nouvelles problématiques sociales? Quelles dimensions prévalent et orientent le travail quotidien? Comment les professionnels se positionnent-ils entre les identités professionnelles de référence ou leur faiblesse dans le cas des professionnels " émergents ", les prescriptions politiques et techniques, les changements organisationnels, le travail prescrit? Selon quelles modalités y parviennent-ils et avec quelles conséquences sur leur vécu professionnel ? Comment perçoivent-ils les autres professionnels intervenant auprès des jeunes ? Et comment se positionnent-ils ? 
Les propositions d'articles peuvent porter sur les professionnels relevant des divers secteurs concernés (socio-éducatif, social, culture, santé, justice, économie, etc.) et exerçant dans des contextes publics et privés. Elles doivent s'attacher à analyser finement les processus de construction des activités et des professionnalités. Sont principalement attendues des propositions d'articles qui privilégient une analyse contrastive ou comparée de ces processus :

- soit en s'intéressant au même type de professionnels dans des contextes d'exercice hétérogènes (variations territoriales, organisationnelles, de mobilisations autour d'un dispositif, d'un public, etc.) ;

- soit à des types de professionnels différents amenés à travailler ensemble ou contraints de le faire ;

- soit à des professionnels « émergents » devant fabriquer leurs activités et construire leur place dans des contextes traditionnellement investis par d'autres métiers.

\section{Bibliographie indicative}

Astier, I. (2000). Droit à l'emploi et magistratures sociales : vers une politique des situations ? Droit et société, 44/45, 143-155.

Avenel C., (2010), Sociologie des quartiers sensibles, Armand Colin, coll. 128, (3 ${ }^{\text {ème }}$ édition)

Broudic J-Y. (2018). Les "bonnes pratiques " à l'épreuve des faits. Du Désir dans le soin et le travail social. Toulouse, Eres.

Brunel, C. (2015). Les diplômes professionnels délivrés par le Ministère de la Ville, de la Jeunesse et des Sports de 2005 à 2013.

Camus, J. (2008). En faire son métier: de l'animation occasionnelle à l'animation professionnelle. Agora débats/jeunesses, (48), 32-44.

Camus, J. (2011). Les cadres sociaux de l'animation en centres de loisirs en France. Pensée plurielle, (26), 25-36.

Camus, J., \& Lebon, F. (2015). Regards sociologiques sur l'animation. Paris : La documentation Française.

Chantraine, G., \& Sallée, N. (2013). «Éduquer et punir ». Travail éducatif, sécurité et discipline en établissement pénitentiaire pour mineurs. Revue française de sociologie, 54(3), 437-464.

Demazière, D., Lessard, C. et Morrissette J. (2013). " Les effets de la Nouvelles Gestion Publique sur le travail des professionnels : transpositions, variations, ambivalences », Education et Sociétés, 32, p. 5-20.

Donzelot Jacques, (2003), Faire société. La politique de la ville aux États-Unis et en France, Seuil, coll. La couleur des idées, 2003

Halpern, C., Lascoumes, P. et Le Galès, P. (dir.) (2014). L'instrumentation de l'action publique. Paris, Presses de la FNSP.

Falchun, T., Robène, L. \& Terret, T. (2016). L'habitus professionnel spécifique aux éducateurs de la protection judiciaire de la jeunesse. Déviance et Société, 40(1), 101-129.

Lebon, F. (2006). Division politique du travail dans l'animation. Agora débats/jeunesses, 40(1), 64-75.

Gilligan, A. (1982). In a different voice: Psychological theory and women's development. Cambridge, MA: Harvard University Press.

Labadie, F. \& Crochu, E. (2018a). Le renforcement des compétences sociales. Un enjeu majeur de la professionnalisation des travailleurs de jeunesse à l'international. Injep Analyses et Synthèses (16).

Labadie, F., \& Crochu, E. (s. d.). (2018b). Les effets des formations destinées aux travailleurs de jeunesse dans le programme européen Erasmus + Jeunesse en action. Rapports d'études $\mathrm{n}^{\circ} 11$.

Lebon, F. (2009). Les animateurs socioculturels. Paris : La Découverte.

Lebon, F. (2014). Portraits de directeurs dans l'animation et dans l'intervention sociale. Empan, (96), 144-150.

Lebon, F., \& Lescure, E. D. (2006). Des «nouvelles professions» entre précarité et flexibilité: animateurs socioculturels et formateurs d'adultes (1982-2002). Regards Sociologiques, (n³2), 83-95.

Lebon, F., \& Lima, L. (2011). Les difficultés au travail dans l'animation. Agora débats/jeunesses, (57), 23-36.

Loncle P., (2011), « La jeunesse au local : sociologie des systèmes locaux d'action publique », Sociologie, vol. 2, $\mathrm{n}^{\circ} 2$, p. 129-147.

Loncle P. (2003) L'action publique malgré les jeunes: les politiques de jeunesse en France de 1870 à 2000 , Paris, coll. Logiques politiques, L'Harmattan. 
Meziani M. (2018). Accompagnements professionnels et socialisations parentales. Des formes de care soumises à leur institutionnalisation. In Guirimand N. Mazereau P. Leplège A. Les nouveaux enjeux du secteur social et médico-social : décloisonner et coordonner les parcours de vie et de soin. Nîmes : Champ Social, 152-164.

Moignard, B. (2018). Les «nouvelles» problématiques éducatives : construction de l'objet. Revue française de pédagogie, 202/1, 65-75.

Moignard B. et Rubi S. (2013). Des dispositifs pour les élèves perturbateurs : les collèges à l'heure de la soustraitance ?. Carrefours de l'éducation, 36, 47-60.

Muniglia V., Thalineau A. (2012). Insertion professionnelle et sociale des jeunes vulnérables. Les conseillers de missions locales entre adaptation et tensions. Politiques sociales et familiales, 108, 73-82.

Poujol, G. (1989). Profession : animateur. Toulouse : Privat.

Paperman, P. Laugier, S. (2005). Le souci des autres : Éthique et politique du Care. EHESS, Paris.

Pattaroni, L. (2005). Le care est-il institutionnalisable ? Quand la politique du care émousse son éthique. In Paperman P. et Laugier S. (Dir.). Le souci des autres : Éthique et politique du Care. EHESS : Paris, 177-203.

Richelle, J.-L., Ziegelmeyer, J.-M. \& Rubi, S. (Éd.) (2013), L'animation socioculturelle professionnelle, quels rapports au politique? Bordeaux : Carrières Sociales Editions, 161-175.

Rosanvallon, P. (1995). La nouvelle question sociale. Repenser l'État-providence. Paris : Seuil.

Ravon, B. \& Ion, J. (2012). Les travailleurs sociaux. Paris : La Découverte.

Sallée, N. (2017). Éduquer sous contrainte. Une sociologie de la justice des mineurs. Paris : EHESS.

Sallée, N. (2014). Des éducateurs dans l'État. Logiques syndicales et identité professionnelle à la Protection judiciaire de la jeunesse. Terrains \& travaux, (25), 75-94.

Tissot, S. (200è). L'État et les quartiers. Genèse d'une catégorie de l'action publique. Paris : Seuil.

Trindade-Chadeau, A. (2012). Faciliter la transition vers l'emploi des jeunes: stratégies locales d'accompagnement. Cahiers de l'action. Paris : Injep, (37).

Tronto, J.-C. (1993). Moral boundaries: A political argument for an ethic of care. New York : Routledge.

Van Zanten Agnès. 2004. Les politiques d'éducation. Paris : PUF.

\section{CALENDRIER}

$1^{\text {er }}$ octobre 2020 : remise des propositions d'articles (1 à 2 pages avec la problématique, la méthodologie et le plan de l'article et une courte notice biographique) à envoyer aux deux coordonnateur.trice.s du dossier

2 novembre 2020 : sélection des propositions d'articles et réponse aux auteur·trice·s

$1^{\text {er }}$ février 2021 : remise des articles

Octobre 2021 : parution du numéro

Les articles (entre 30000 et 35000 signes) préciseront la problématique, les données empiriques mobilisées, le cadre dans lequel l'étude a été menée, la méthodologie employée et les résultats obtenus.

\section{COORDINATION DU NUMERO}

Valérie Becquet : valerie.becquet@cyu.fr

Martial Meziani : martial.meziani@cyu.fr

Revue Agora débats/jeunesses agora@injep.fr

Rédactrices en chef : Yaëlle Amsellem Mainguy et Marianne Autain

0170989419 / 0170989434 